\title{
Random effects tumour growth models for identifying image markers of mammography screening sensitivity
}

https://doi.org/10.1515/em-2019-0022

Received June 17, 2019; accepted August 26, 2020; published online September 15, 2020

\begin{abstract}
Introduction: Percentage mammographic density has long been recognised as a marker of breast cancer risk and of mammography sensitivity. There may be other image markers of screening sensitivity and efficient statistical approaches would be helpful for establishing them from large scale epidemiological and screening data.

Methods: We compare a novel random effects continuous tumour growth model (which includes a screening sensitivity submodel) to logistic regression (with interval vs. screen-detected cancer as the dependent variable) in terms of statistical power to detect image markers of screening sensitivity. We do this by carrying out a simulation study. We also use continuous tumour growth modelling to quantify the roles of dense tissue scatter (measured as skewness of the intensity gradient) and percentage mammographic density in screening sensitivity. This is done by using mammograms and information on tumour size, mode of detection and screening history from 1,845 postmenopausal women diagnosed with invasive breast cancer, in Sweden between 1993 and 1995.

Results: The statistical power to detect a marker of screening sensitivity was larger for our continuous tumour growth model than it was for logistic regression. For the settings considered in this paper, the percentage increase in power ranged from 34 to $56 \%$. In our analysis of data from Swedish breast cancer patients, using our continuous growth model, when including both percentage mammographic density and dense tissue scatter in the screening sensitivity submodel, only the latter variable was significantly associated with sensitivity. When included one at a time, both markers were significantly associated (p-values of $5.7 \times 10^{-3}$ and $1.0 \times 10^{-5}$ for percentage mammographic density and dense tissue scatter, respectively).

Conclusions: Our continuous tumour growth model is useful for finding image markers of screening sensitivity and for quantifying their role, using large scale epidemiological and screening data. Clustered dense tissue is associated with low mammography screening sensitivity.
\end{abstract}

Keywords: breast cancer tumour growth; dense tissue scatter; mammographic density; random effects; screening sensitivity.

\section{Introduction}

In order to evaluate and develop screening programmes for breast cancer it is important to understand mammography screening sensitivity. The fact that tumours can be masked at screening if they are hidden in mammographically dense tissue has long been known (Whitehead et al. 1985). Dense tissue hides tumours

\footnotetext{
*Corresponding author: Linda Abrahamsson, Department of Medical Epidemiology and Biostatistics, Karolinska Institute, Box 281 , 17177 Stockholm, Sweden, E-mail: blekingestatistik@gmail.com. https://orcid.org/0000-0002-1372-5508

Maya Alsheh Ali, Kamila Czene, Gabriel Isheden and Keith Humphreys, Department of Medical Epidemiology and Biostatistics, Karolinska Institute, Stockholm, Sweden, E-mail: maya.alsheh.ali@ki.se (M. Alsheh Ali), kamila.czene@ki.se (K. Czene), gabriel.isheden@ki.se (G. Isheden), keith.humphreys@ki.se (K. Humphreys)

Per Hall, Department of Medical Epidemiology and Biostatistics, Karolinska Institute, Stockholm, Sweden; Department of Oncology, Södersjukhuset, Stockholm, Sweden, E-mail: per.hall@ki.se
}

Ә Open Access. ( 2020 Linda Abrahamsson et al., published by De Gruyter. $(\Subset)$ BY This work is licensed under the Creative Commons Attribution 4.0 International License. 
because both tumours and dense (fibroglandular) tissues appear white on mammograms (non-dense tissues [fat] appear dark). Other techniques, such as magnetic resonance imaging, ultrasonography and digital breast tomosynthesis have a higher sensitivity in mammographically dense breasts, but these are more costly (Freer 2015). As well as affecting mammography screening sensitivity, mammographic density (MD) is a wellestablished, strong breast cancer risk factor (Boyd et al. 2007).

Over the last decades MD has often been assessed using either percentage mammographic density (PD) or absolute mammographic density. PD measures the fraction of pixels of a mammogram which appear light (above a certain threshold). There exist both semi-automated (Byng et al. 1994) and fully automated procedures (Li et al. 2012) for measuring PD, and mammography screening sensitivity has been quantified as a continuous function of PD and tumour size (Abrahamsson and Humphreys 2016). PD however does not take into account how the dense tissue might be distributed in the breast.

The Breast Imaging Reporting and Data System, BI-RADS (D’Orsi et al. 2013), is used as a guideline for radiologists to visually assess MD into four different categories (a. the breasts are almost entirely fatty; $b$. there are scattered areas of fibroglandular density; c. the breasts are heterogeneously dense, which may obscure small masses; d. the breasts are extremely dense, which lowers the sensitivity of mammography). In an earlier version of BI-RADS, from 2003, categories were constructed to also include information on overall PD ( $<25 \%$; $25-50 \%$; 50-75\%; $>75 \%)$. Subsequent changes to BI-RADS ensured that women with a small amount of PD, but located in a way that could mask tumours, are now classified with breast composition c, instead of b. PD alone does not explain the differences in screening sensitivities across different women.

In order to demonstrate that MD is associated with mammographic sensitivity several researchers have compared interval and screen-detected cancers, e.g. Mandelson et al. (2000). Interval cancers are those detected (symptomatically) between scheduled screens, in women regularly attending screening, and overrepresent tumours missed at previous screens (as well as fast growing tumours and tumours with a nondelayed symptomatic detection). Strand et al. (2016) tested 32 different image features of MD tissue for association with interval versus screen-detected cancer (after adjusting for PD). There was one feature, capturing how scattered the dense tissue is, which had a statistically significant association with interval/ screening cancer status, which was suspected to be attributable to a relationship with screening sensitivity. We revisit this feature in this paper.

In addition to being studied in the context of risk of interval cancer, image features have been studied before for their role in breast cancer risk, see for example Häberle et al. (2012), Zheng et al. (2015) and Eriksson et al. (2017). Other than PD, image features have not been studied directly for their role in mammography screening sensitivity, using natural history/tumour growth models, which is what we do here.

A continuous tumour growth modelling framework, such as the one we describe in section "Continuous tumour growth modelling", can be applied to breast cancer screening data for quantifying the role of image markers of sensitivity and even for revealing new image markers. The term "continuous growth model" refers to the fact that tumour size is considered on a continuous scale in the underlying latent model of growth, and distinguishes these models from multi-state models (Uhry et al. 2010), where tumours pass through discrete states of pre-clinical and clinical cancer. In the current article we aim to, firstly, compare the statistical power of logistic regression (comparing interval versus screen-detection status) to that of using a continuous tumour growth model, for finding associations between image markers and screening sensitivity. We do so by conducting a simulation study (sections "Simulation study to assess the statistical power to detect markers" and "Simulation study"). Secondly, using data from Swedish breast cancer patients, we directly quantify the relationship between dense tissue scatter and screening sensitivity by fitting a continuous growth model; see sections "Quantification of screening sensitivity in terms of tumour size, PD and dense tissue scatter using observational study data" under section "Methods and materials" and "Quantification of screening sensitivity in terms of tumour size, PD and dense tissue scatter using observational study data" under section "Results". 


\section{Methods and materials}

\section{Logistic regression of interval/screen-detection status}

Researchers have used population based studies and compared interval and screen-detected cases of breast cancer (using logistic regression), to find markers of tumour aggressiveness (Holm et al. 2015) and screening sensitivity (Krishnan et al. 2016; Mandelson et al. 2000). They have modelled the log odds for being an interval case as

$$
\log \left(\frac{\pi}{1-\pi}\right)=\alpha_{0}+\boldsymbol{\alpha}_{1} \mathbf{x}
$$

where $\pi=P$ (interval case) and $1-\pi=P$ (screen-detected case), $\boldsymbol{\alpha}_{1}=\left(\alpha_{11}, \ldots, \alpha_{1 \mathrm{p}}\right)$ is a vector of coefficients and $\mathbf{x}^{\prime}=\left(\mathrm{x}_{1}, \ldots, \mathrm{x}_{\mathrm{p}}\right)$ is a covariate vector (containing e.g. image markers, such as $\mathrm{PD}$ ). Although logistic regression is an easy-to-implement and fast procedure, in the context of comparing interval and screen-detected cancer patients, as explained in the introduction, it only indirectly addresses associations with sensitivity because detection mode is determined through several processes (screening sensitivity, tumour growth rate and symptomatic detectability).

\section{Continuous tumour growth modelling}

In this paper we examine an alternative approach to finding (and quantifying the role of) markers of screening sensitivity, which is based on fitting a continuous tumour growth model. The proposed model enables all breast cancer patients, not only those that are screen attenders, to be included in analyses, as long as information on detection mode, screening history, tumour size and mammographic images is available. We use the continuous tumour growth model proposed in Abrahamsson and Humphreys (2016), together with an estimation procedure described in Isheden and Humphreys (2019). The model consists of three submodels; for tumour growth, time to symptomatic detection and mammography screening sensitivity. Parameter estimation is based on modelling the conditional probabilities of tumour size at diagnosis, given screening history, detection mode (symptomatic/screening) and any covariates included in the submodels for tumour growth, symptomatic sensitivity and screening sensitivity (Isheden and Humphreys 2019). The modelling approach essentially treats symptomatic and screen-detection as competing events. Through incorporating tumour growth as a latent process, our approach incorporates the negative results of previous screens. Tumour sizes are determined probabilistically at screens under the parametric modelling assumptions described above. This is done via conditional distributions of growth rates, given tumour sizes at detection (for a complete description of the conditional growth rate distributions see Isheden and Humphreys 2019). Through the likelihood estimation procedure, we sum over conditional distributions of tumour sizes at prior negative screens, for both symptomatic and screen-detected tumours and simultaneously use tumour sizes of screen-detected tumours to model screening sensitivity.

In previous publications we have described simulation studies to check that values of model parameters can be accurately retrieved using our maximum likelihood procedure (Appendix of Isheden et al. 2019). In our approach, we do not model death as a competing event. This will have a small impact on the estimation of growth rate distributions, as discussed in Isheden and Humphreys (2019). This point applies equally well to the estimation of sojourn times using the widely applied (semi-) Markov multi-state models for cancer screening (Uhry et al. 2010).

Submodel for screening sensitivity: The most important submodel for this study is that which describes screening sensitivity. The model allows the probability of a tumour to be detected at screening to be a logistic function of its size, as well as characteristics of the breast images (such as PD or other image markers). The approach we use essentially incorporates screening sensitivity at all screening occasions using tumour size at diagnosis for the positive screen (for screen-detected cases) and the probabilistically infered tumour sizes at prior negative screens (for both screening and symptomatic cases with previous negative screens). A logistic form has also been used by Weedon-Fekjær et al. (2008) to model sensitivity within a continuous modelling framework. When considering covariates/markers, in addition to tumour diameter in $\mathrm{mm}$ at the time of screening, denoted here as $d$, the probability for a tumour to be detected by the radiologist at a mammogram (i.e. the sensitivity of mammography screening) is

$$
\frac{\exp \left(\boldsymbol{\beta}_{\mathbf{0}} \mathbf{z}+\boldsymbol{\beta}_{1} \mathbf{x}\right)}{1+\exp \left(\boldsymbol{\beta}_{0} \mathbf{z}+\boldsymbol{\beta}_{1} \mathbf{x}\right)}
$$

where $\mathbf{z}^{\prime}=(1, d), \boldsymbol{\beta}_{\mathbf{0}}=\left(\beta_{00}, \beta_{01}\right)$ and $\boldsymbol{\beta}_{\mathbf{1}}=\left(\beta_{11}, \ldots, \beta_{1 \mathrm{p}}\right)$ are vectors of coefficients. As before, $\mathbf{x}$ is a $(\boldsymbol{p} \times 1)$ vector of covariates/ markers. Both (1) and (2) are logistic functions, but (2) models screening sensitivity directly (as opposed to interval/screen detection status) and, unlike (1), (2) includes (latent) tumour size as a covariate.

Submodel for tumour growth: Tumours are assumed to be spherical and to grow exponentially. Each tumour has an individual constant volume doubling time (until the time point of detection). The volume (in $\mathrm{mm}^{3}$ ) for a tumour, growing with an inverse 
growth rate $r, t$ years after tumour onset (which we define here as the point at which a tumour is $0.5 \mathrm{~mm}$ in diameter - a size at which a tumour can be reasonably assumed to be detectable and from which it will grow deterministically), is specified as

$$
V(t, r)=V_{0} e^{t / r}, \quad t \geq 0, r>0,
$$

where $V_{0}$ is the volume corresponding to a tumour diameter of $0.5 \mathrm{~mm}$. To allow for heterogeneous growth rates between tumours, the inverse growth rate, $r$, is modelled as a random variable, $R$, assumed to follow a gamma distribution, with shape and rate parameters $\tau_{1}$ and $\tau_{2}$. The density function for $R$ is

$$
f_{R}(r)=\frac{\tau_{2}^{\tau_{1}}}{\Gamma\left(\tau_{1}\right)} r^{\tau_{1}-1} e^{-\tau_{2} r}, r \geq 0
$$

with mean and variance being $E(R)=\tau_{1} / \tau_{2}$ and $\operatorname{Var}(R)=\tau_{1} / \tau_{2}^{2}$. This model has been used before (Bartoszyński et al. 2001; Plevritis et al. 2007) and it has been shown to lead to mathematical tractable results (Isheden and Humphreys 2019). In particular, the distribution of tumour sizes at symptomatic detection (in the absence of screening) has a closed form. If we had instead assumed that growth rate (as opposed to inverse growth rate) is gamma distributed, this is not the case. Under our assumptions, the distribution of growth rate does not have a common distributional form. Importantly, the exponential growth has been shown by Talkington and Durrett (2015) to give a good fit to breast cancer in vivo data.

Submodel for symptomatic detection: We model time to symptomatic detection, $T_{\text {det }}$, from tumour onset, using a tumour volume (calculated according to formula (3)) dependent hazard (instantaneous risk) function

$$
h_{T_{\text {det }}}(t)=\eta V(t, r), V(t, r) \geq V_{0},
$$

where $\eta$ is a constant. This submodel has also been used in previous studies (Bartoszyński et al. 2001; Plevritis et al. 2007). Volume at symptomatic detection will of course be unobserved if a tumour is detected through screening.

Likelihood estimation: For parameter estimation we divide tumour diameters into small intervals. We let $p_{i, j}$ be the (conditional) probability for a tumour in woman $j$ to be detected in size interval $i$, and $o_{i, j}$ be an indicator function equal to 1 if woman $j$ has a tumour detected in size interval $i$, and 0 otherwise. The likelihood of the set of observations given a parameter set $\theta$ is

$$
L(\mathbf{o} \mid \boldsymbol{\theta})=\prod_{j} \prod_{i} p_{i, j}{ }^{o_{i, j}},
$$

The matrix $\mathbf{o}$ consists of all values $o_{i, j} \cdot \boldsymbol{\theta}=\left(\tau_{1}, \tau_{2}, \eta, \beta_{00}, \beta_{01}, \beta_{11}, \ldots, \beta_{1 p}\right)$ is a vector of the submodel parameters (the number of parameters depending on how many variables/factors are included in the sensitivity submodel). The discretisation of tumour size is appropriate since (some) pathologists can round off tumour size values. It has also been shown, through simulation, that this discretisation results in negligible bias (Isheden and Humphreys 2019; Isheden et al. 2019).

The probabilities $p_{i, j}$ are conditioned on detection mode, dates of previous negative screenings, and (image) marker values. Isheden and Humphreys (2019) have derived expressions for these probabilities, using the three submodels for tumour growth, symptomatic detection and screening sensitivity. For self-containment, a shortened description of the procedures for calculating $p_{i, j}$ are provided in the Appendix. Calculations of $p_{i, j}$ are based on the assumption of a stable disease population - which requires that the rate of births in the population, the distribution of age at tumour onset and the distribution of time to symptomatic detection are constant across calendar time. For practical purposes this assumption will be reasonable as long as the data being analysed is gathered from cancer patients diagnosed within a relatively short time interval (several years).

Computational practicalities: We use the following procedures for parameter estimation in all analyses based on our tumour growth model. We include a maximum number of three earlier negative screens in the calculations, to ease computations. Tumour diameters in $\mathrm{mm}$ (recorded from pathologists reports in our observational study as the maximum distance across the tumour, recorded after surgery, and from the diagnosis date in our simulation), are categorised into the following intervals (in mm); [0,1.5), $[1.5,2.5),[2.5,7.5),[7.5,12.5),[12.5,17.5), \ldots,[67.5,72.5),[72.5,85),[85,95),[95,105), \ldots,[145,155]$. Maximisation of the likelihood function (6), was carried out using the modified quasi-Newton optimisation procedure in the optim function in the statistical program R (2011) (using the option $L-B F G S-B$ ).

\section{Simulation study to assess the statistical power to detect markers of screening sensitivity}

We carry out a simulation study to compare the statistical power of using a continuous tumour growth model to that of using logistic regression (comparing interval vs. screen-detected cancer), for detecting markers of screening sensitivity. A summary of the main simulation study setup is provided in Table 1. In our main simulation, we generated 500 cohorts, each of which contained 
approximately 1,900-2,000 breast cancer cases between the ages 40 and 74. For each cohort we generated (in-silico) individuals being born at a constant rate over a long time period and, after allowing for a time, of 150 years, sufficient to achieve burn-in (i.e. a constant incidence of cancers with an appropriate mix of tumour growth rates), we collected incident (interval and screen-detected) cancers over a time period of two years.

Age-specific breast cancer incidence rates from The Swedish National Board of Health and Welfare (according to rates from 2016-accessed 2018), subsequently shifted with five years towards lower ages, were used to generate an age at tumour onset, in a similar manner to Forastero et al. (2010). The value of five years was close to the mean value of time between tumour onset and detection in the main simulation. For all individuals we simulated a time of death from other causes, according to Swedish agespecific mortality rates (values of 2016-accessed 2018) from Statistics Sweden, so that they could be removed if they died from other causes before being diagnosed with breast cancer.

Using the exponential growth model (3)-(4), each woman (independent of tumour onset time point) was assigned an inverse tumour growth rate sampled from a gamma distribution with shape and rate parameter values of $\tau_{1}=2.09$ and $\tau_{2}=4.36$ (Isheden and Humphreys 2019). Under this continuous tumour growth model, in combination with the hazard function (5), there exists a closedform conditional distribution for symptomatic tumour size, $V_{\text {det }}$, given inverse tumour growth rate. Using the cdf of this distribution

$$
F_{V \mid R=r}(v)=1-\exp \left(-\eta r\left(v-V_{0}\right)\right), \quad v \geq V_{0},
$$

we could sample a (conditional) symptomatic tumour size for each woman. We used a parameter value of $\eta=e^{-8.04}$ (from Isheden and Humphreys 2019). Then, by using Eq. (3), on the basis of a calendar time point of tumour onset, an inverse tumour growth rate and a symptomatic tumour size, each woman could be assigned a calendar time point for symptomatic detection in the absence of screening.

All women between the ages 40 and 74 attend screening. This made it possible to include the same set of women when using the different approaches to analysis (sections "Logistic regression of interval/screen-detection status" and "Continuous tumour growth modelling”) - the logistic regression approach cannot deal with symptomatic cases that are not interval

Table 1: Simulation study comparing the ability of the methods of logistic regression and continuous tumour growth modelling to detect image markers affecting mammography screening sensitivity.

Simulation input

Simulation output

Method of estimation

Variables included

in estimation method

Estimation details
Constant birth rate into cohort over 150 years

Swedish age-specific breast cancer incidence rates

Swedish age-specific mortality rates

Tumours growing with an exponential growth function with gamma distributed inverse growth rates

Hazard function for time to symptomatic detection dependent on tumour size

Screening programme for all women aged $40-74$ years old

Complete screen attendance every second year

Time-independent image marker from a standard normal distribution

Logistic function for screening sensitivity dependent on tumour size and image marker

500 cohorts, each having 1,900-2,000 incident breast cancer cases aged 40-74 years old
Logistic regression model

Dependent variable: Detection mode

Independent variable: Image marker

\author{
Continuous tumour growth model \\ Detection mode \\ Image marker \\ Tumour size \\ Negative screening history \\ Joint estimation of \\ Mammography screening sensitivity \\ (as a function of image marker and tumour size) \\ Tumour growth \\ Time to symptomatic detection
}

Statistical power of 500 likelihood ratio tests for inclusion of image marker in model 
cancers. Following an initial screen, women were screened every second year, up until cancer detection, age 75 or death, whichever came first.

For each woman a simulated marker value was generated from a standard normal distribution, and was assumed to be constant across time. At each screen occasion, the screening sensitivity was calculated using the logistic function (2), using parameter values $\beta_{00}=-5.01$ (intercept), $\beta_{01}=0.58$ (for tumour size at screening) and $\beta_{11}=-0.25$ (for the marker). The values of $\beta_{00}$ and $\beta_{01}$ were chosen based on a previous data analysis (Isheden and Humphreys 2019). The value of $\beta_{11}$ was chosen such that it enabled comparisons across a relevant range of statistical powers.

We calculated statistical power as the probability of obtaining a significant association at a significance level; of $\alpha=0.05$. For both continuous tumour growth model estimation (association of the image marker with screening sensitivity) and the logistic regression model (association of the image marker with interval/screen-detected cancer) we used likelihood ratio tests, i.e. we compared nested models, with/without the marker included in the model.

The main simulation was repeated for a range of inverse tumour growth rate distributions, with mean inverse growth rate $E(R)=\tau_{1} / \tau_{2}$. In addition to a mean inverse growth rate of 0.48 , which has previously been estimated from observational data (Isheden and Humphreys 2019) we used values of 0.25, 0.3, 0.35, 0.4, 0.6, 0.75 and 1 . These values correspond to mean doubling times of 2.1, 2.5, 2.9, 3.3, 5.0, 6.2 and 8.3 months. The different distributions were simulated by varying the value of the parameter $\tau_{2}$.

As a check of our estimation procedure we also studied type-I error and checked distributions of parameter estimates. We note that we have also previously performed thorough checks of our estimation procedure, using simulation (Isheden et al. 2019). To check type-I error we simulated 500 cohorts, (with $E(R)=0.48$ ) using the same procedure as for our power calculations, but fixing $\beta_{11}=0$.

For a sensitivity analysis, we also explored a scenario where the assumption of an exponential-gamma tumour growth model used in the estimation procedure was incorrect, by instead simulating under an alternative tumour growth submodel, in which tumours were assumed to grow according to a logistic function

$$
V(t)=\frac{V_{\max }}{\left[1+\left(\left(\frac{V_{\max }}{V_{\text {cell }}}\right)^{0.25}-1\right) e^{-0.25 k t}\right]^{4}},
$$

where $t$ is the time from tumour onset in years, $\kappa$ is the growth rate, $V_{\text {cell }}$, is the volume of one cell, $V_{\text {max }}$, is the assumed maximum volume (corresponding to a tumour diameter of $128 \mathrm{~mm}$ ). The growth rate $\kappa$ was assumed to be a realisation from the lognormal distribution with mean and variance parameters $\mu$ and $\sigma^{2}$. This model of screening sensitivity has also been used by Weedon-Fekjær et al. (2008). We used parameter values of $\mu=1.03$ and $\sigma^{2}=1.31$ (Weedon-Fekjær et al. 2008) for the lognormally growth rate distribution. The other submodels (for symptomatic detection and screening sensitivity) used the same parameter values as were used in the main simulation. To obtain calendar time point for symptomatic detection we divided time into small intervals of length $d t \approx 4$ days. For each interval, $t$, we assumed the probability to be detected by symptoms to be $P=\eta V_{t} d t$, where $V_{t}$ is the volume of the tumour in interval $t$ (retrieved by using the mid time point of the interval). With small intervals, we could, obtain a good approximation to the hazard function (5), across time, when using the logistic-lognormal growth model.

The focus of our simulation study is on whether or not there is a difference in statistical power between the continuous growth and logistic regression methods due to how they handle the processes of screening sensitivity, tumour growth and symptomatic detectability (combined within detection mode in the logistic regression and modelled explicitly in the continuous tumour growth model). This is why we simulated screening with complete attendance. We know that in practice there will be an additional advantage for the continuous tumour growth modelling approach, due to its ability to include also non-regular attenders of screening and women diagnosed before reaching the age at which screening starts to be offered.

\section{Dense tissue scatter and screening sensitivity}

As described in section "Introduction", Strand et al. (2016) tested 32 different image features of MD tissue for association with interval versus screen-detected cancer (after adjusting for PD), using logistic regression. Two of the features were reported to be associated. One of these, skewness of the intensity gradient, was hypothesised to affect screening sensitivity (the other, eccentricity, was suggested to be associated with symptomatic tumour detectability). The authors speculated that skewness of the intensity gradient, reflects the amount of fatty streaks that are intermixed with the dense area - a low value corresponds to scattered dense tissue and an increased screening sensitivity (a more technical description of the feature is provided in section "Quantification of screening sensitivity in terms of tumour size, PD and dense tissue scatter using observational study data" under section "Methods and materials"). Although their arguments appear reasonable, based on their logistic regression analysis it was not possible to show concretely how the feature is related to screening sensitivity. Figure 1 displays two mammographic images with similar PD and total 
breast area values (measured in pixels), but with markedly different values of the feature. It is possible that a radiologist would allocate these mammograms to different BI-RADS categories.

Strand et al. (2016) validated the association between this feature and detection mode in an external data set. They presented a $95 \%$ confidence interval for the odds ratio (per standard deviation change) of $(1.04,1.41)$ after adjusting for PD and other factors, which corresponds to a p-value of 0.014. In the section following we use the same study population (as that used in the validation study in Strand et al. (2016)), but re-analyse the data using our random effects tumour growth model.

\section{Quantification of screening sensitivity in terms of tumour size, PD and dense tissue scatter using observational study data}

We used observational data to study the impact of two image markers, PD and dense tissue scatter (skewness of the intensity gradient), on screening sensitivity. We analysed data from postmenopausal incident primary invasive breast cancer patients, diagnosed in Sweden between October 1993 and March 1995, collected as part of a case-control study (Magnusson et al. 1998). This case-control study had a participation rate of $84 \%$ among the 3,979 cases initially deemed to be eligible through Swedish Cancer Registries. Of the 3,345 participating cases, 698 had to be removed because of not meeting the inclusion criteria, the reasons being noninvasive breast cancer, the diagnosed breast cancer not being the primary one, diagnosis occurring outside the study period, the woman being premenopausal (or below the age of 55 for women with missing information on menopausal status) or that the woman had received neoadjuvant treatment.

Exclusions also had to be made due to lack of mammogram, or missing information on cause for diagnostic mammogram, i.e. screening or symptomatic, tumour size and screening history. Lack of screening history was defined as women reporting in questionnaires that they had been screened, when no such records could be found in the screening history data. For women having a recorded screening, a contralateral mammogram (using the mediolateral-oblique view) was searched for. Contralateral mammograms were used to that image marker values would be unaffected by the presence of tumours; both MD and tumours appear white on mammograms. We included women that had at least one such mammogram within up to seven years prior to diagnosis. We ended up with a final data set of 1,845 patients. The steps of data exclusion can be followed in Figure 2. In summary, of all of the exclusions, 802 were excluded due to missing data on at least one of the variables used in statistical analyses. For 200 of these, the detection mode was missing, 60 had missing information on tumour size, 265 were missing screening history and 277 had no available mammogram. For the 602 excluded cases having information on detection mode, 52\% were screen-detected, compared to the $61 \%$ of the included cases in this study. Of the included 1,845 patients, 1,124 were screen-detected and 721 were symptomatically detected (of which 390 cases had a tumour diagnosed within the regular screening interval of two years from a negative screening). Collection of the data, including the retrieval of analogue mammographic images and screening histories from Swedish mammography screening units and radiology departments has been described previously in detail (Abrahamsson et al. 2015; Eriksson et al. 2012; Rosenberg et al. 2008).

From the mammographic images we retrieved a measure of PD calculated by an automatised method (which uses the program ImageJ) which has been validated by Li et al. (2012) against the user-assisted Cumulus method (Eriksson et al. 2012). We note that symptomatically detected cases without a previous negative screening do not need to have image marker values to be included in our analysis based on our continuous growth model (our data set included 205 such women). Since there were

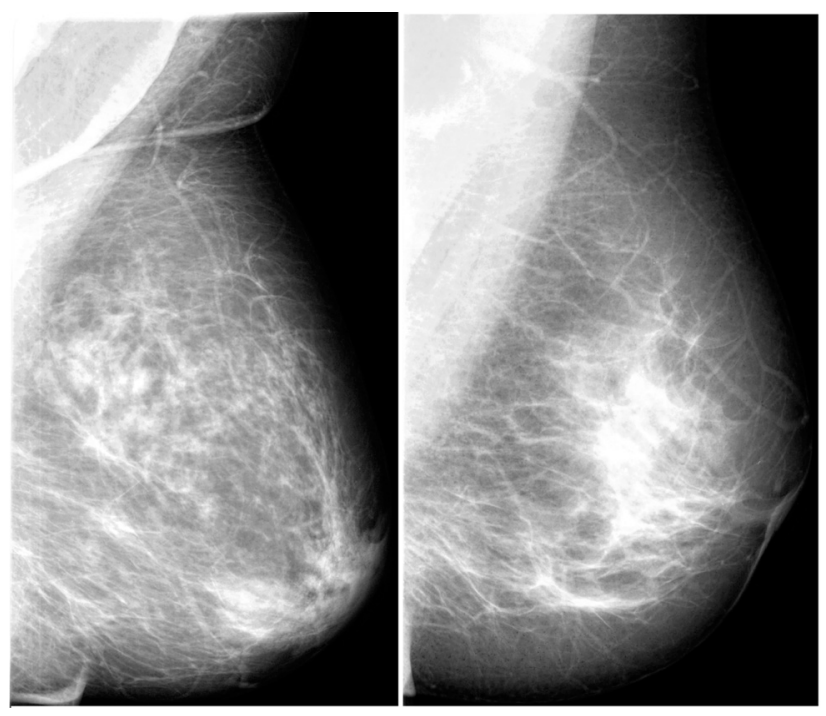

Figure 1: An example of a difference in skewness of the intensity gradient, with low skewness (scattered MD) represented on the left mammogram and high skewness (clustered MD) represented on the right mammogram. Images from two women with similar percentage mammographic densities (around 20\%) and similar total breast area values. 


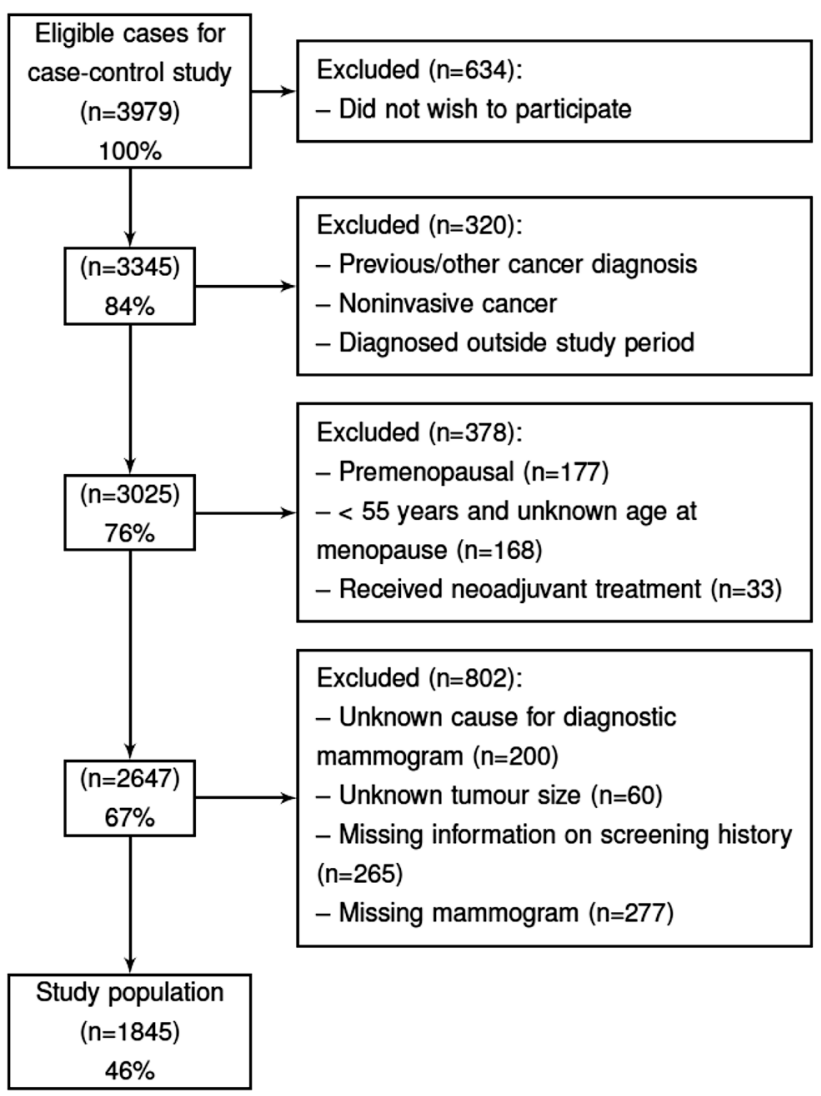

Figure 2: Exclusion steps in preparation of the study population used to estimate mammography screening sensitivity as a function of tumour size, percentage mammographic density and dense tissue scatter.

many women for which some, but not all, images were retrieved, we used a single PD-value for each woman, which was calculated as the average value based on all her included (i.e. recent pre-diagnostic) mammograms. It has been shown that PD decreases with age (Boyd et al. 2002), however we assume that the decrease over the (maximum of 7) years from which we include mammograms, will not be large enough to affect our parameter estimates to any noticeable extent. We also measured skewness of the intensity gradient from the same set of mammograms, again, taking the mean value of several mammograms per woman.

The image feature (skewness of the intensity gradient extracted from the dense area) was extracted from the mammograms by applying three processing steps (pre-processing of the image (including reducing differences in intensity contrast between mammograms and removal of the pectoral muscle), selection of dense area and extraction of feature) which have been explained in depth previously (Strand et al. 2016). For the dense area selection we used Otsu's thresholding technique (Otsu 1979) which has been widely used for segmenting mammographic images. MATLAB (2017) was used for calculating the feature values.

More technically, skewness of the intensity gradient explains the difference in pixel intensities between two neighbouring pixels (on the horizontal direction for simplification). All pixels outside the dense area were set as having a zero intensity. The largest difference between pixel intensity (gradient) will thus be around the border of the dense region (as chosen by the thresholding technique). There will however also be a difference between pixels inside the dense area, but this difference will be smaller. Between pixels in non-dense areas the difference will be zero. The two main types of differences (zero or not) will lead to a bimodal shape of the distribution of the intensity gradient. The skewness of the distribution will be larger when the dense area is homogeneous and not interspersed by fatty streaks, due to having more differences with value zero in relation to differences between dense-area border and interior pixels.

As mentioned earlier, for some women, images were not retrieved from all registered (negative) screens. For PD, we used one single value per woman (the average over all included mammograms). To aid interpretation of skewness of the intensity gradient (SI) we constructed a normalised version of skewness, $0 \leq \mathrm{SI}^{\star} \leq 1$, such that $\mathrm{SI}^{\star}=\frac{\mathrm{SI}-\min (\mathrm{SI})}{\max (\mathrm{SI})-\min (\mathrm{SI})}$, where $\min (\mathrm{SI})$ and $\max (\mathrm{SI})$ represent the minimum and maximum mean values for SI, which are -0.57 and 2.11 .

Based on the continuous tumour growth model estimation (section "Continuous tumour growth modelling") we quantified the role of PD and our normalised version of skewness of the intensity gradient, SI*, in the screening sensitivity function (2) by adding a covariate for $\mathrm{SI}^{\star}$, so that, in (2), $\mathbf{z}=(1, d)$, as before, and $\mathbf{x}=(m, s)$, where $d$ is the tumour diameter in $\mathrm{mm}$ at screening (an observed variable for positive screens and a latent variable for negative screens), $m(0 \leq m \leq 1)$ is $\mathrm{PD}$ and $s(0 \leq s \leq 1)$ is SI*. We call 
this model, which in total consists of seven parameters (including two parameters for the growth rate model (3)-(4) and one for the symptomatic detection function (5)), Model D. In total we fitted four different models, which differed only in terms of the screening sensitivity function; in addition to tumour size, Model A includes no image markers, Model B includes PD only and Model C includes SI* only. We compared the four models using likelihood ratio tests. For each model, to obtain variability estimates for the parameters, we used 200 non-parametric bootstrap samples and the percentile method (to calculate $95 \%$ coverage intervals).

We worked under the assumption that PD and $\mathrm{SI}^{\star}$ do not affect symptomatic detection or tumour growth rate. Oestricher et al. (2002) did not find evidence for an effect of MD (measured with BI-RADS) on clinical breast examination sensitivity. Also, in an earlier study, no strong evidence was found for MD being associated with Ki-67, a marker for tumour proliferation (Heusinger et al. 2012).

Ethical approval for the study was obtained from the Regional Ethics Review Board in Uppsala at Uppsala University and ethical approvals of extensions of the study were obtained from the Regional Ethics Review Board in Stockholm at Karolinska Institutet. Written informed consent was provided from all participants.

Data simulation procedure to exemplify image marker quantification: To illustrate the ability of the image markers PD and SI* to separate women into groups of low/high screening sensitivity (which may not be clear from examining the parameter estimates and $\log$ likelihood values) we carried out a small simulation study on masking. We made use of two different simulations, first based on the parameter estimates of Model B and second based on those of Model C (we did not simulate under Model D as it turned out not to fit significantly better than Model C; see section "Quantification of screening sensitivity in terms of tumour size, PD and dense tissue scatter using observational study data” under section "Results”). Apart from being based on different parameter estimates, the setups were the same for both simulations. We defined the group of women that have a tumour (of $5 \mathrm{~mm}$ or more) present on at least one screen, as the screen detection-eligible group. We defined masking as an event when a tumour of at least $5 \mathrm{~mm}$ in diameter is missed at a screening. Furthermore, we calculated risk ratios of being masked, among women in the screen detection-eligible group, comparing groups of women having high and low values of either PD, or SI ${ }^{\star}$, determined by upper and lower quartiles in the respective simulation.

Each simulation included 250,000 breast cancer cases. The simulation procedure was the same as that presented in section "Simulation study to assess the statistical power to detect markers of screening sensitivity", except that the single simulated image marker from a normal distribution was replaced, first by PD (for simulation based on Model B) and then by SI* (for simulation based on Model C), in order to mimic real data distributions. PD was simulated from a beta distribution with shape parameters of 1.66 and 7.82 , and $\mathrm{SI}^{\star}$ was simulated as the logarithm of values from a normal distribution with $\mu=1.88$ and $\sigma=0.26$. These distributions were estimated from real data (see section "Quantification of screening sensitivity in terms of tumour size, PD and dense tissue scatter using observational study data”) using the function fitdistr in R (2011).

\section{Results}

\section{Simulation study}

In Figure 3 we plot empirical estimates of statistical power to detect association of the simulated marker with screening sensitivity/detection mode for both the continuous tumour growth model (dashed line) and the logistic regression model (solid line). The statistical power is presented for different rates of inverse tumour growth, $R$, presented as $1 / E(R)=\tau_{2} / \tau_{1}$ so that growth rate is faster to the right side of the plot. Observe that $1 /$ $E(R)$ is not equal to the mean tumour growth rate due to the skewed appearance of the inverse tumour growth rate distribution (4). In all cases the continuous tumour growth model estimation had higher power than the logistic regression one. For both models, power decreases with increased rates of tumour growth. When tumours grow faster they are more likely to be detected symptomatically, so this result is to be expected. The fractions of screen-detected cases (in percentages) were 64, 59, 55, 51, 47, 45, 41, 37, going from the slowest growing tumours to the fastest growing ones, in the different simulation setups. This is however not the only reason for the power decrease. When tumours grow faster, there are fewer tumours present at previous negative screens, which will also lead to a decrease in power, at least for the continuous tumour growth model estimation.

We note that, when simulating under $\beta_{11}=0$ (section "Simulation study to assess the statistical power to detect markers of screening sensitivity"), likelihood ratio tests rejected the null hypothesis of no association between marker and sensitivity, in exactly 5\% (25) of the 500 cohorts. The mean and standard error of the 


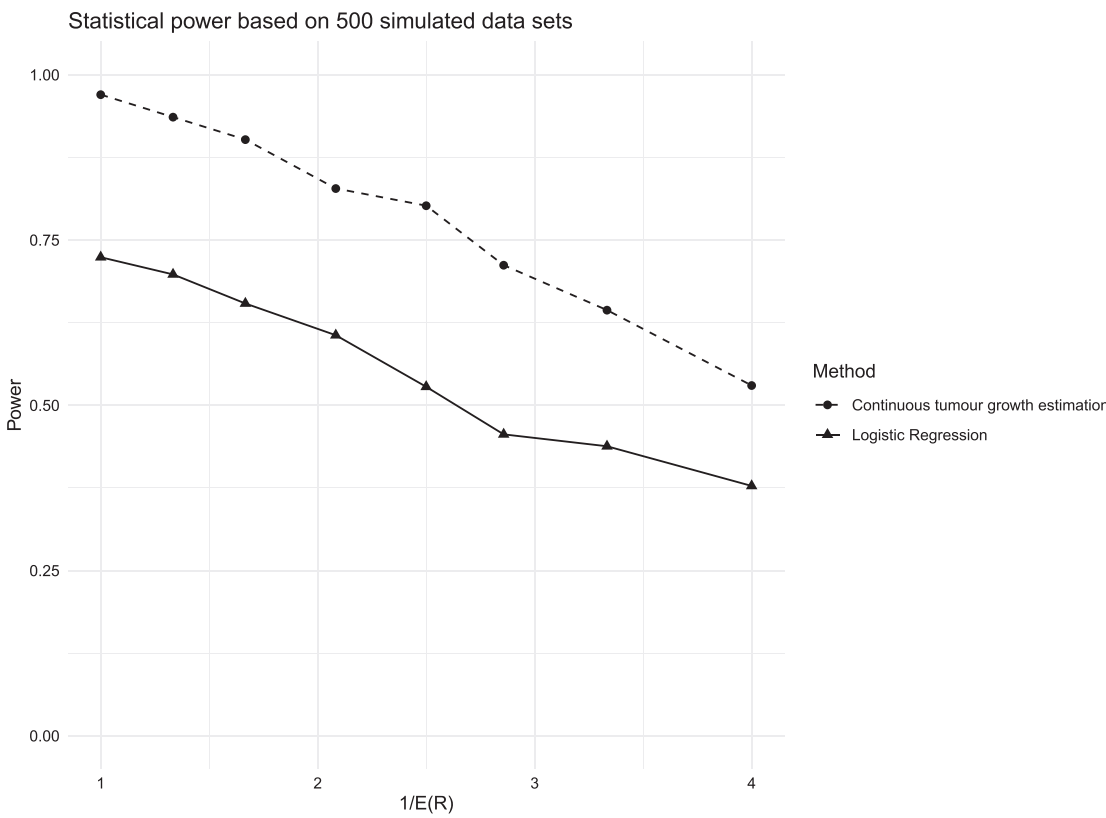

Figure 3: Statistical power for detecting image markers associated with screening sensitivity, using continuous tumour growth estimation versus using logistic regression for different tumour growth rate distributions.

estimates of $\beta_{11}$ were 0.0001 and 0.006 . The percentage bias in the estimate of $\beta_{01}$ (tumour size) was $1.34 \%$. For the simulated cohorts with $\beta_{11}=-0.25$ (and $E(R)=0.48$ ) the percentage biases in $\beta_{01}$ and $\beta_{11}$ were 0.64 and $0.58 \%$. This demonstrates that although some approximations are involved in our model (categorising tumour size), as has been shown previously (Isheden et al. 2019), parameters are estimated with very little bias.

To investigate the impact of assuming an incorrect tumour growth model, we generated data on 500 data sets of approximately 2,000 cases using the logistic-lognormal tumour growth model setting presented in Weedon-Fekjær et al. (2008) (section "Quantification of screening sensitivity in terms of tumour size, PD and dense tissue scatter using observational study data" under section "Methods and materials"). This gave rise to a $79.0 \%$ power for the continuous tumour growth model and a $57.8 \%$ power for the logistic regression. Although we cannot make a direct comparison to the results obtained under the exponential growth model (e.g. the rate of growth changes over time for the logistic growth model), the most relevant point to compare results to is where the logistic regression model has the same power (apx. 58\%). Reading from Figure 2, at this point, the correct (exponential) growth model obtained a power of just over $80 \%$.

\section{Quantification of screening sensitivity in terms of tumour size, PD and dense tissue scatter using observational study data}

To quantify the role of dense tissue scatter (and PD) in mammography screening sensitivity we made use of the data on incident postmenopausal breast cancer cases described in section "Quantification of screening sensitivity in terms of tumour size, PD and dense tissue scatter using observational study data" under section "Methods and materials". To exemplify the impact of dense tissue scatter on masking we carried out a small simulation study (section "Data simulation procedure to exemplify image marker quantification").

Key characteristics of the data are presented in Table 2. Of the 1,640 women having mammograms (recall that 205 women had symptomatically detected cancer, and no previous screen - these women do not need information on features in order to be included in the estimation procedure), the first and third quartiles of the number of included mammograms were 3 and 5. There was a strong correlation (Spearman correlation coefficient of 0.77) between (average) PD and (average) SI. A scatter plot of the variables is shown in Figure 4.

In Table 3 we present parameter estimates and 95\% non-parametric bootstrap coverage intervals based on fitting Models A, B, C and D. Maximum log likelihood values of the models are shown in Table 4. Likelihood 
Table 2: Descriptive comparison of screen- and symptomatically detected cases.

\begin{tabular}{lrr}
\hline & $\begin{array}{r}\text { Screen-detected } \\
\text { cases }\end{array}$ & $\begin{array}{r}\text { Symptomatically } \\
\text { detected cases }\end{array}$ \\
\hline Number of cases & 1,124 & 721 \\
Cases with no previous negative screening & 130 & 205 \\
Cases with 1 previous negative screening & 211 & 98 \\
Cases with 2 previous negative screenings & 658 & 244 \\
Cases with 3 previous negative screenings & 125 & 174 \\
Time since last negative screening in years; $\quad(1.82,2.13,11.57 \%)$ & 1.38 \\
$\quad$ median (quartiles and \% NA) & 63 & 2.01 \\
Age at diagnosis; $\quad(58,68)$ & 12 & 63 \\
$\quad$ median (quartiles) & $(9,18)$ & $(57,70)$ \\
$\quad$ Tumour size, mm; & 14.01 & 20 \\
$\quad$ median (quartiles) & $(13,26)$ \\
Percentage mammographic density; $\quad$ median (quartiles and \% missing) & $17.97,28.43 \%)$ \\
$\quad$ Normalised skewness of the intensity gradient; & $(7.77,22.21,0 \%)$ & 17.38 \\
$\quad$ median (quartiles and \% missing) & $(0.52,0.71,0 \%)$ & 0.65 \\
\hline
\end{tabular}

ratio tests, in turn comparing Models $B$ and $C$ to Model A, give rise to $p$-values of $5.7 \times 10^{-3}$ (for PD) and $1.0 \times 10^{-5}$ (for $\mathrm{SI}^{\star}$ ). Comparing, in turn, Models B and C to Model D, in order to evaluate the effect of each marker, independent of the other, gives rise to $\mathrm{p}$-values of 0.76 (for PD) and $5.6 \times 10^{-4}$ (for SI*). In a sensitivity analysis we used median values of PD and SI*, instead of mean values for the mammograms. Parameter estimates were very similar to those in Table 3.

Based on the simulation described in section "Data simulation procedure to exemplify image marker quantification”, we calculated risk ratios of being masked with a tumour larger than $5 \mathrm{~mm}$ in diameter (among women in the screen detection-eligible group), comparing groups of women having different PD and SI*; see Table 5. Women with a high PD or SI* (i.e. falling in the upper quartile in the respective simulation based on Model B or Model C), had a higher risk of having been masked, compared to women falling in the lower quartiles of PD or SI* (risk ratios of 1.20 and 1.38). There are fewer women in the lower quartile of $\mathrm{SI}^{\star}$ that are

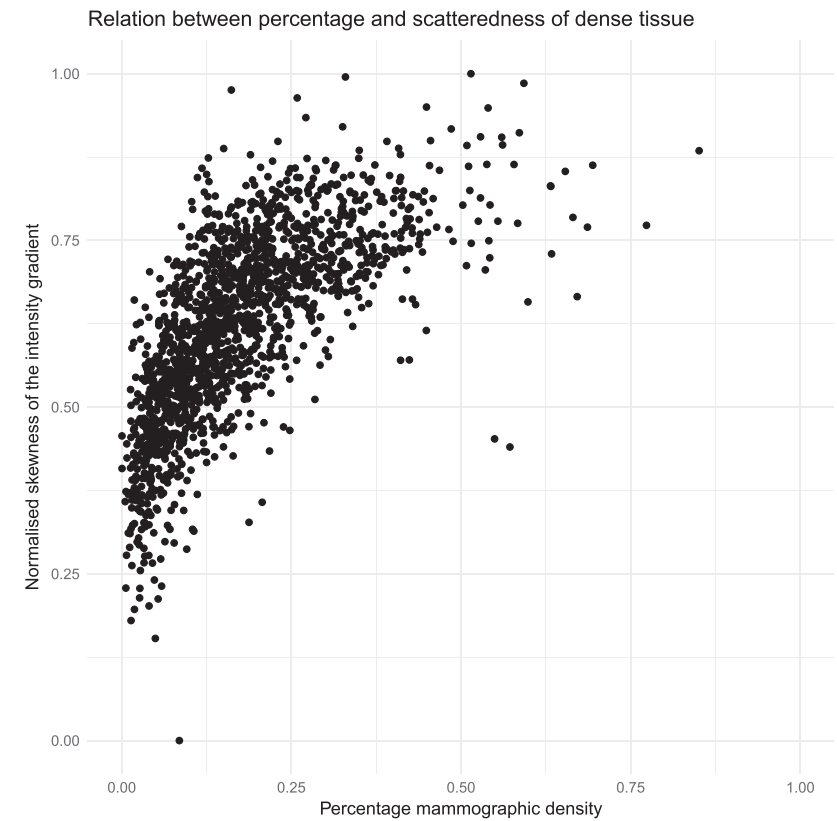

Figure 4: Dense tissue scatter (measured as normalised skewness of the intensity gradient) in relation to percentage mammographic density, measured from analogue mammographic images of Swedish postmenopausal breast cancer cases. 
Table 3: Parameter estimates, presented with $95 \%$ non-parametric bootstrap coverage intervals, of screening sensitivity, tumour growth and time to symptomatic detection for the Swedish postmenopausal breast cancer data.

\begin{tabular}{|c|c|c|c|c|}
\hline Parameter & Model A & Model B & Model C & Model D \\
\hline \multicolumn{5}{|l|}{ Submodel for screening sensitivity } \\
\hline \multirow{2}{*}{ Intercept $\left(\beta_{00}\right)$} & -4.99 & -4.66 & -3.37 & -3.32 \\
\hline & $(-6.16,-4.77)$ & $(-5.11,-4.37)$ & $(-4.34,-2.53)$ & $(-4.31,-2.45)$ \\
\hline \multirow{2}{*}{ Tumour size $\left(\beta_{01}\right)$} & 0.58 & 0.57 & 0.59 & 0.59 \\
\hline & $(0.51,0.99)$ & $(0.51,0.73)$ & $(0.51,0.73)$ & $(0.51,0.72)$ \\
\hline \multirow{2}{*}{$\begin{array}{l}\text { Percentage mammographic } \\
\text { density }\left(\beta_{11}\right)\end{array}$} & & -2.14 & & 0.34 \\
\hline & - & $(-4.03,-0.46)$ & - & $(-2.01,2.58)$ \\
\hline \multicolumn{2}{|l|}{ Normalised skewness of } & & -2.76 & -2.94 \\
\hline the intensity gradient $\left(\beta_{12}\right)$ & - & - & $(-4.31,-1.49)$ & $(-4.91,-0.89)$ \\
\hline \multicolumn{5}{|l|}{ Submodel for tumour growth } \\
\hline \multirow[t]{2}{*}{ Shape parameter $\left(\tau_{1}\right)$} & 2.10 & 2.08 & 2.11 & 2.12 \\
\hline & $(1.61,2.86)$ & $(1.61,2.77)$ & $(1.62,2.82)$ & $(1.63,2.82)$ \\
\hline \multirow[t]{2}{*}{ Rate parameter $\left(\tau_{2}\right)$} & 4.45 & 4.27 & 4.32 & 4.35 \\
\hline & $(3.17,8.22)$ & $(2.95,6.83)$ & $(3.06,6.58)$ & $(3.04,6.54)$ \\
\hline \multirow{2}{*}{ Mean inverse growth rate $\left(\frac{\tau_{1}}{\tau_{2}}\right)$} & 0.47 & 0.49 & 0.49 & 0.49 \\
\hline & $(0.29,0.65)$ & $(0.31,0.67)$ & $(0.33,0.67)$ & $(0.33,0.67)$ \\
\hline \multicolumn{5}{|c|}{ Submodel for time to symptomatic detection } \\
\hline \multirow[t]{2}{*}{ Intercept $(-\log (\eta))$} & 8.04 & 8.08 & 8.09 & 8.08 \\
\hline & $(7.54,8.49)$ & $(7.57,8.50)$ & $(7.58,8.51)$ & $(7.58,8.50)$ \\
\hline
\end{tabular}

masked, in comparison to those in the lower quartile of PD. SI* improves separation of women into groups of high/low sensitivity.

\section{Discussion}

In this article we have proposed the use of a continuous tumour growth model for detecting mammographic image markers related to mammography screening sensitivity. We have shown that statistical power is increased, in comparison to using logistic regression with detection mode as the outcome variable (interval versus screening case), when the same set of women is included when applying both methods. Although there are more parameters to estimate in the continuous tumour growth model, it has the advantage that it separates the latent process of screening sensitivity from processes of tumour growth rate and symptomatic detectability. In observational studies the power advantage will be even larger, since women that are not regular attenders of screening can be included in the continuous growth model approach (as long as information on screening attendance (and on images) is available). In the simulation study, the statistical power was shown to vary according to growth rate distribution. However the percentage decrease in power from using logistic regression was fairly constant across different mean inverse growth rates. We have also shown that the power difference

Table 4: Maximum log likelihood values for models including no image marker, PD only, SI* only or both PD and $\mathrm{SI}^{\star}$, in the screening sensitivity function.

\begin{tabular}{lrr}
\hline Model & $\begin{array}{r}\text { Maximum } \\
\text { Log likelihood }\end{array}$ & $\begin{array}{r}\text { Number of } \\
\text { Estimated parameters }\end{array}$ \\
\hline Model A: $\beta_{11}=0, \beta_{12}=0$ & -3562.68 & 5 \\
Model B: $\beta_{11} \neq 0, \beta_{12}=0$ & -3558.86 & 6 \\
Model C: $\beta_{11}=0, \beta_{12} \neq 0$ & -3552.94 & 6 \\
Model D: $\beta_{11} \neq 0, \beta_{12} \neq 0$ & -3552.90 & 7 \\
\hline
\end{tabular}


Table 5: Risk ratios of being masked, comparing women with high to low levels (quartiles) of image markers, based on simulated data.

\begin{tabular}{lllll}
\hline $\begin{array}{l}\text { Model } \\
\text { simulated } \\
\text { from }\end{array}$ & Image marker & $\begin{array}{l}\text { No. of women } \\
\text { with TS }>\mathbf{5 ~} \mathbf{~ m m} \\
\text { on at least 1 screen }\end{array}$ & $\begin{array}{l}\text { No. of women } \\
\text { ever } \\
\text { masked with TS > 5 } \mathbf{~ m m}\end{array}$ & $\begin{array}{l}\text { Risk ratio of } \\
\text { being masked }\end{array}$ \\
\hline Model B & PD (upper vs. lower quartile) & $39,713,38,603$ & $21,109,17,105$ & 1.20 \\
Model C & SI* (upper vs. lower quartile) & $39,976,38,040$ & $21,436,14,773$ & 1.38 \\
\hline
\end{tabular}

between the methods is still substantial when the underlying tumour growth model assumption of exponential growth is wrong (by simulating under the logistic-lognormal tumour growth model). Thus for the purpose of detecting image markers related to screening sensitivity the power advantage is likely to outweigh any wrong modelling assumption. There exist other approaches for modelling the natural history of breast cancer. The multi-state Markov model (Uhry et al. 2010) has been widely used to analyse cancer screening cohort data. In this article we have focused on developing a continuous growth model because, firstly, it has been developed for our study design (for the analysis of data from incident cases in a population based case-control study) and, secondly, it is closer to the underlying biology than the multi-state Markov model, as it incorporates separate processes of tumour growth, symptomatic detection and screening sensitivity.

We have shown that our continuous growth model has a higher power than the logistic regression to detect features affecting screening sensitivity. However, our model should be used carefully and our calculations are carried out under the assumption that the markers are solely associated with screening sensitivity, and not with tumour growth rate or rate of symptomatic detection. In Strand et al. (2016), 32 features were tested for association with mode of detection, and two features were reported as being associated: skewness of the intensity gradient, SI, and eccentricity. According to Strand et al. (2016), only the first feature would be clear to have an effect on screening sensitivity per se. The assumption that SI is independent of symptomatic detection and tumour growth rate is likely to be reasonable and there is a strong hypothesis of association with sensitivity for SI (Strand et al. 2016). Since the other features in Strand et al. (2016) were not discussed in depth there, we have not carried out similar analyses for these. Our calculations of statistical power in the current paper, were, similarly, made under the assumption that the marker was not associated with the other processes (tumour growth, symptomatic detection).

In our simulation in section "Simulation study to assess the statistical power to detect markers of screening sensitivity", as stated above, our image marker was associated only with screening sensitivity and not symptomatic sensitivity or tumour growth rate. For the continuous growth model it would be possible to isolate effects on sensitivity by including the marker as a covariate also in the growth and symptomatic detection models. Although computational times are being improved upon (Isheden and Humphreys 2019), adding parameters would of course make the estimation procedure more time-consuming, especially for bootstrapping procedures.

We have have presented new results concerning the role of dense tissue scatter in screening sensitivity, i.e. that clustered dense tissue is more likely to mask a tumour than scattered dense tissue. Results from this study suggest that skewness of the intensity gradient is a superior predictor of mammography screening sensitivity and that PD adds no independent information about sensitivity, given this feature. However, PD is a wellestablished measure of sensitivity (and is additionally associated with breast cancer risk (Boyd et al. 2007)). Our finding thus needs to be replicated in larger studies, possibly also on premenopausal women. In this study mammographic density was only retrieved from analogue images. It would be interesting to validate its association with screening sensitivity using digital images.

With the simulation study presented in section "Quantification of screening sensitivity in terms of tumour size, PD and dense tissue scatter using observational study data" under section "Results" regarding the ability of PD versus dense tissue scatter to determine which women that have high or low screening sensitivity, we showed that the risk ratio of masking, comparing upper to lower quartiles of dense tissue scatter, is larger than 
for PD. The numbers serve as a good illustration of the importance of the marker in masking/sensitivity, but the exact magnitude of its effect should be viewed with caution since the simulation is based on some simplifications (e.g. all women participate in screening with 100\% attendance) and not all parameter values are estimated from real data (e.g. time at tumour onset).

Whilst dense tissue scatter/skewness of the intensity gradient appears to be an important image marker for screening sensitivity, in future studies it would be interesting to test other relevant image markers for association with screening sensitivity, using our continuous tumour growth model framework.

\section{Conclusion}

Through a simulation study we have shown that random effects tumour growth modelling is a powerful tool for finding relevant image features from mammograms important for screening sensitivity. It has the advantage of being able to separate the effects of screening sensitivity, tumour growth rate and symptomatic detection. Using observational data we have shown that skewness of the intensity gradient, as a marker of dense tissue scatter, in comparison to percent density (PD), improves our ability to separate women into groups having low or high mammography screening sensitivity. Understanding how mammographic density lowers mammography screening sensitivity may help decisions about which women are in need of other techniques for screening than mammography.

Research funding: This work was supported by the Swedish Research Council (2016-01245), the Swedish Cancer Society (grant CAN 2017/287) and the Swedish e-Science Research Centre. KC was supported by Stockholm County Council (grant number 20170088).

Author contributions: All authors have accepted responsibility for the entire content of this manuscript and approved its submission.

Competing interests: The authors declare that they have no competing interests.

Informed consent: Informed consent was obtained from all individuals included in this study.

\section{Appendix: Calculation of conditional probabilities of tumour size, $\boldsymbol{p}_{i, j}$}

We summarise here the calculation of the conditional probabilities of tumour size, conditional on patients' detection modes and screening histories. A more complete description is provided in Isheden and Humphreys (2019). We use the same notation as they do. In that article, a description of the underlying stable disease population assumptions, under which calculations are performed, are spelled out. We use these assumptions and the following random variables:

- $A(s)=1$ if an individual has a symptomatically undetected tumour at time point $s$, $A(s)=0$ if otherwise, at time point $s$.

- $B(s)=1$ if a tumour is detected by screening at time point $s$, $B(s)=0$ if no tumour is detected by screening at time point $s$.

- $\quad C(s) \in[0, \infty)$ : the size of the tumour at time point $s$.

- $\quad D(s)=1$ if a tumour is symptomatically detected at time point $s$, $D(s)=0$ if no tumour is symptomatically detected at time point $s$.

In deriving the conditional probabilities (below), we omit subscript $j$ (i.e. we write $p_{i}$ instead of $p_{i, j}$ ).

\section{A1 Screen-detected tumours}

For screen-detected tumours, we write the probability for a tumour to be in size interval $I_{i}$ (the 24 size intervals we use are defined in section "Computational practicalities") as 


$$
p_{i}=P\left(C(s) \in I_{i} \mid A(s)=1, B(s)=1, \mathbf{B}^{\mathbf{c}}\right),
$$

where $s$ is time of diagnosis, $\mathbf{B}^{\mathbf{c}}$ denotes that $\left(B\left(s_{-k}\right), \ldots, B\left(s_{-1}\right)\right)=(0, \ldots, 0)$ and $s_{-k}, \ldots, s_{-1}$ represent the (ordered) times of the $k$ previous (negative) screens $\left(s_{-1}\right.$ representing the most recent negative screen before diagnosis). Then, $p_{i}$ can be written as

$$
\begin{aligned}
& p_{i} \propto P\left(B(s)=1 \mid C(s) \in I_{i}\right) P\left(C(s) \in I_{i} \mid A(s)=1\right) \\
& \cdot \sum_{l \leq i} P\left(\mathbf{B}^{\mathbf{c}} \mid C(s) \in I_{i}, C\left(s_{-1}\right) \in I_{l}\right) P\left(C\left(s_{-1}\right) \in I_{l} \mid C(s) \in I_{i}\right) ; \quad k \geq 1 \\
& p_{i} \propto P\left(B(s)=1 \mid C(s) \in I_{i}\right) P\left(C(s) \in I_{i} \mid A(s)=1\right) ; \quad k=0
\end{aligned}
$$

for $i=1, \ldots, 24$ and $l=0, \ldots, i$, where $I_{0}$ represents a state prior to tumour initiation and $I_{1}$ has $V_{0}$ as its lower limit. The term $P(B(s)=1 \mid C(s) \in I)$ is the screening sensitivity, which is specified using the logistic function; Eq. (2) in main text. $P\left(C(s) \in I_{i} \mid A(s)=1\right)$ is the probability that the tumour is in size interval $I_{i}$, given the presence of an undetected tumour. Isheden and Humphreys (2019) derive a closed form for this expression under the assumption of a stable disease population. This can be obtained as a function of the distribution of tumour volumes in the absence of screening (see Plevritis et al. 2007), which we denote here as $P\left(V_{\text {det }} \in I_{i}\right)$.

The probability for having negative results at the previous screens is assumed to be

$$
P\left(\mathbf{B}^{\mathbf{c}} \mid C(s) \in I_{i}, C\left(s_{-1}\right) \in I_{l}\right)=P\left(B\left(s_{-1}\right)=0\right) \cdot P\left(B\left(s_{-2}\right)=0\right) \cdots P\left(B\left(s_{-k}\right)=0\right),
$$

where $P(B(t)=0)=1-P(B(t)=1) . P(B(t)=1)$ is the probability that a tumour is found at screening, as a function of tumour size at time $t$ and the mammographic image markers (again, Eq. (2) in main text). Tumour sizes at $s_{-k}, \ldots s_{-2}$ are obtained probabilistically by projecting backwards from the trajectory intersecting the midpoints of intervals $I_{i}$ and $I_{j}$.

The probability for (unobserved) tumour size at the most recent negative screen conditional on tumour size (at diagnosis), $P\left(C\left(s_{-1}\right) \in I_{l} \mid C(s) \in I_{i}\right)$, is calculated by marginalising over the conditional growth rate distribution (for details, see Eqs. (7) and (9), and accompanying text, in Isheden and Humphreys 2019).

\section{A2 Symptomatically detected tumours}

For symptomatically detected cases (with $k$ previous negative screens),

$$
p_{i}=P\left(C(s) \in I_{i} \mid \mathbf{B}^{\mathbf{c}}, D(s)=1\right),
$$

and

$$
\begin{gathered}
p_{i} \propto P\left(V_{\text {det }} \in I_{i}\right) \\
\cdot \sum_{l \leq i} P\left(\mathbf{B}^{\mathbf{c}} \mid C\left(s_{-1}\right) \in I_{l}, C(s) \in I_{i}, D(s)=1\right) P\left(C\left(s_{-1}\right) \in I_{l} \mid C(s) \in I_{i}, D(s)=1\right) ; \quad k \geq 1, \\
p_{i} \propto P\left(V_{\text {det }} \in I_{i}\right) ; \quad k=0 .
\end{gathered}
$$

As mentioned above, $P\left(V_{\mathrm{det}} \in I_{i}\right)$ can be obtained using the distribution of tumour volumes in the absence of screening. $P\left(\mathbf{B}^{\mathbf{c}} \mid C\left(s_{-1}\right) \in I_{l}, C(s) \in I_{i}, D(s)=1\right)$ is obtained by backwards projection in the same way as for the screen-detected case (see A1), and $P\left(C\left(s_{-1}\right) \in I_{l} \mid C(s) \in I_{i}, D(s)=1\right)$ is obtained using the conditional cumulative density function for the growth rate conditioning on the size at symptomatic detection (Eq. (7) in Isheden and Humphreys 2019).

As long as screening attendance is independent of growth rate and other assumptions are valid, this likelihood based procedure obtains unbiased estimates of all parameters in the sub-models ((2), (4) and (5); main text). This assumption will be reasonable in samples of postmenopausal women diagnosed with breast cancer, i.e. as in the observational study described in section "Quantification of screening sensitivity in terms of tumour size, PD and dense tissue scatter using observational study data”. 


\section{References}

Abrahamsson, L., K. Czene, P. Hall, and K. Humphreys. 2015. "Breast Cancer Tumour Growth Modelling for Studying the Association of Body Size with Tumour Growth Rate and Symptomatic Detection Using Case-Control Data." Breast Cancer Research 17: 116.

Abrahamsson, L., and K. Humphreys. 2016. "A Statistical Model of Breast Cancer Tumour Growth with Estimation of Screening Sensitivity as a Function of Mammographic Density." Statistical Methods in Medical Research 25: 1620-37.

Bartoszyński, R., L. Edler, L. Hanin, A. Kopp-Schneider, L. Pavlova, A. Tsodikov, A. Zorin, and A. Y. Yakovlev. 2001. "Modeling Cancer Detection: Tumor Size as a Source of Information on Unobservable Stages of Carcinogenesis." Mathematical Biosciences 171: 113-42.

Boyd, N., L. Martin, J. Stone, L. Little, S. Minkin, and M. Yaffe. 2002. "A Longitudinal Study of the Effects of Menopause on Mammographic Features." Cancer Epidemiology and Prevention Biomarkers 11: 1048-53.

Boyd, N. F., H. Guo, L. J. Martin, L. Sun, J. Stone, E. Fishell, R. A. Jong, G. Hislop, A. Chiarelli, S. Minkin, and M. J. Yaffe 2007. "Mammographic Density and the Risk and Detection of Breast Cancer." New England Journal of Medicine 356: 227-36.

Byng, J. W., N. Boyd, E. Fishell, R. Jong, and M. J. Yaffe. 1994. "The Quantitative Analysis of Mammographic Densities." Physics in Medicine \& Biology 39: 1629.

D’Orsi, C., E. Sickles, E. Mendelson, and E. Morris. 2013. ACR BI-RADS Atlas: Breast Imaging Reporting and Data System. Reston (VA): American College of Radiology.

Eriksson, L., K. Czene, L. Rosenberg, K. Humphreys, and P. Hall. 2012. "The Influence of Mammographic Density on Breast Tumor Characteristics." Breast Cancer Research and Treatment 134: 859-66.

Eriksson, M., K. Czene, Y. Pawitan, K. Leifland, H. Darabi, and P. Hall. 2017. "A Clinical Model for Identifying the Short-Term Risk of Breast Cancer." Breast Cancer Research 19: 29.

Forastero, C., L. Zamora, D. Guirado, and A. Lallena. 2010. "A Monte Carlo Tool to Simulate Breast Cancer Screening Programmes.” Physics in Medicine \& Biology 55: 5213.

Freer, P. E. 2015. “Mammographic Breast Density: Impact on Breast Cancer Risk and Implications for Screening.” Radiographics 35: 302-15.

Häberle, L., F. Wagner, P. A. Fasching, S. M. Jud, K. Heusinger, C. R. Loehberg, A. Hein, C. M. Bayer, C. C. Hack, M. P. Lux, K. Binder, M. Elter, C. Münzenmayer, R. Schulz-Wendtland, M. Meier-Meitinger, B. R. Adamietz, M. Uder, M. W. Beckmann, and T. Wittenberg. 2012. “Characterizing Mammographic Images by Using Generic Texture Features.” Breast Cancer Research 14: R59.

Heusinger, K., S. M. Jud, L. Häberle, C. C. Hack, P. A. Fasching, M. Meier-Meitinger, M. P. Lux, C. Hagenbeck, C. R. Loehberg, T. Wittenberg, C. Rauh, F. Wagner, M. Uder, A. Hartmann, R. Schulz-Wendtland, M. W. Beckmann, D. L. Wachter. 2012. "Association of Mammographic Density with the Proliferation Marker Ki-67 in a Cohort of Patients with Invasive Breast Cancer." Breast Cancer Research and Treatment 135: 885-92.

Holm, J., K. Humphreys, J. Li, A. Ploner, A. Cheddad, M. Eriksson, S. Törnberg, P. Hall, and K. Czene. 2015. “Risk Factors and Tumor Characteristics of Interval Cancers by Mammographic Density." Journal of Clinical Oncology 33: 1030-7.

Isheden, G., L. Abrahamsson, T. Andersson, K. Czene, and K. Humphreys. 2019. "Joint Models of Tumour Size and Lymph Node Spread for Incident Breast Cancer Cases in the Presence of Screening." Statistical Methods in Medical Research 28: 3822-42.

Isheden, G., and K. Humphreys. 2019. “Modelling Breast Cancer Tumour Growth for a Stable Disease Population.” Statistical Methods in Medical Research 28: 681-702.

Krishnan, K., L. Baglietto, C. Apicella, J. Stone, M. C. Southey, D. R. English, G. G. Giles, and J. L. Hopper. 2016. “Mammographic Density and Risk of Breast Cancer by Mode of Detection and Tumor Size: a Case-Control Study." Breast Cancer Research 18: 63.

Li, J., L. Szekely, L. Eriksson, B. Heddson, A. Sundbom, K. Czene, P. Hall, and K. Humphreys. 2012. "High-throughput Mammographic-Density Measurement: A Tool for Risk Prediction of Breast Cancer.” Breast Cancer Research 14: R114.

Magnusson, C., J. Baron, I. Persson, A. Wolk, R. Bergström, D. Trichopoulos, and H.-O. Adami. 1998. “Body Size in Different Periods of Life and Breast Cancer Risk in Post-menopausal Women." International Journal of Cancer 76: 29-34.

Mandelson, M. T., N. Oestreicher, P. L. Porter, D. White, C. A. Finder, S. H. Taplin, and E. White. 2000. "Breast Density as a Predictor of Mammographic Detection: Comparison of Interval-And Screen-Detected Cancers." Journal of the National Cancer Institute 92: 1081-7.

MATLAB. 2017, Version 9.3. Natick: The MathWorks Inc.

Oestreicher, N., E. White, C. D. Lehman, M. T. Mandelson, P. L. Porter, and S. H. Taplin. 2002. "Predictors of Sensitivity of Clinical Breast Examination (CBE)." Breast Cancer Research and Treatment 76: 73-81.

Otsu, N. 1979. “A Threshold Selection Method from Gray-Level Histograms.” IEEE Transactions on Systems, Man, and Cybernetics 9: 62-6.

Plevritis, S. K., P. Salzman, B. M. Sigal, and P. W. Glynn. 2007. “A Natural History Model of Stage Progression Applied to Breast Cancer." Statistics in Medicine 26: 581-95.

R Development Core Team. 2011. R: A Language and Environment for Statistical Computing. Vienna, Austria: R Foundation for Statistical Computing. https://www.r-project.org.

Rosenberg, L. U., F. Granath, P. W. Dickman, K. Einarsdóttir, S. Wedrén, I. Persson, and P. Hall. 2008. “Menopausal Hormone Therapy in Relation to Breast Cancer Characteristics and Prognosis: A Cohort Study.” Breast Cancer Research 10: R78. 
Statistics Sweden. 2018. Statistical Database on Mortality. Available from https://www.statistikdatabasen.scb.se (accessed October 22, 2018).

Strand, F., K. Humphreys, A. Cheddad, S. Törnberg, E. Azavedo, J. Shepherd, P. Hall, and K. Czene. 2016. “Novel Mammographic Image Features Differentiate between Interval and Screen-Detected Breast Cancer: A Case-Case Study.” Breast Cancer Research 18: 100.

Talkington, A., and R. Durrett. 2015. “Estimating Tumor Growth Rates In Vivo.” Bulletin of Mathematical Biology 77: $1934-54$.

The National Board of Health and Welfare. 2018. Statistics Database for Cancer. Available from https://www.socialstyrelsen.se/ statistik/statistikdatabas/cancer (accessed October 22, 2018).

Uhry, Z., G. Hédelin, M. Colonna, B. Asselain, P. Arveux, A. Rogel, C. Exbrayat, C. Guldenfels, I. Courtial, P. Soler-Michel, F. Molinié, D. Eilstein, and S. W. Duffy. 2010. “Multi-state Markov Models in Cancer Screening Evaluation: A Brief Review and Case Study." Statistical Methods in Medical Research 19: 463-86.

Weedon-Fekjær, H., B. H. Lindqvist, L. J. Vatten, O. O. Aalen, and S. Tretli. 2008. "Breast Cancer Tumor Growth Estimated through Mammography Screening Data." Breast Cancer Research 10: R41.

Whitehead, J., T. Carlile, K. J. Kopecky, D. J. Thompson, F. I. Gilbert Jr., A. J. Present, B. A. Threatt, P. Krook, and E. Hadaway. 1985. "Wolfe Mammographic Parenchymal Patterns. A Study of the Masking Hypothesis of Egan and Mosteller." Cancer 56: 1280-6.

Zheng, Y., B. M. Keller, S. Ray, Y. Wang, E. F. Conant, J. C. Gee, and D. Kontos. 2015. "Parenchymal Texture Analysis in Digital Mammography: A Fully Automated Pipeline for Breast Cancer Risk Assessment.” Medical Physics 42: 4149-60. 